\title{
On wavemodes at the interface of a fluid and a fluid-saturated poroelastic solid
}

\author{
K. N. van Dalen, ${ }^{\text {a) }}$ G. G. Drijkoningen, and D. M. J. Smeulders \\ Department of Geotechnology, Faculty of Civil Engineering and Geosciences, Delft University \\ of Technology, Stevinweg 1, 2628 CN Delft, The Netherlands
}

(Received 21 April 2009; revised 12 January 2010; accepted 12 January 2010)

\begin{abstract}
Pseudo interface waves can exist at the interface of a fluid and a fluid-saturated poroelastic solid. These waves are typically related to the pseudo-Rayleigh pole and the pseudo-Stoneley pole in the complex slowness plane. It is found that each of these two poles can contribute (as a residue) to a full transient wave motion when the corresponding Fourier integral is computed on the principal Riemann sheet. This contradicts the generally accepted explanation that a pseudo interface wave originates from a pole on a nonprincipal Riemann sheet. It is also shown that part of the physical properties of a pseudo interface wave can be captured by loop integrals along the branch cuts in the complex slowness plane. Moreover, it is observed that the pseudo-Stoneley pole is not always present on the principal Riemann sheet depending also on frequency rather than on the contrast in material parameters only. Finally, it is shown that two additional zeroes of the poroelastic Stoneley dispersion equation, which are comparable with the $\bar{P}$-poles known in nonporous elastic solids, do have physical significance due to their residue contributions to a full point-force response.
\end{abstract}

(C) 2010 Acoustical Society of America. [DOI: 10.1121/1.3308473]

PACS number(s): 43.35.Pt, 43.20.Jr, 43.20.Gp, 43.20.Bi [JM]

Pages: 2240-2251

\section{INTRODUCTION}

Interface waves such as Rayleigh and Stoneley waves are often used to investigate materials. One can think of applications in ultrasonic testing of structures, borehole logging in geotechnical and reservoir engineering, and surface seismics in geophysics, see, e.g., Refs. 1-4. In the case of porous materials, interface waves carry information on elastic properties but also on properties such as porosity, permeability, and fluid mobility. ${ }^{1}$ Rosenbaum ${ }^{5}$ found that, compared to all other surface and body wavemodes, the Stoneley-type wave that travels along the open-pore interface of a fluid and a porous medium, carries the best measure of permeability.

Several theoretical studies were performed on interface waves that propagate along the boundary of a porous medium. These studies were carried out in the context of Biot's theory for wave propagation in fluid-saturated poroelastic solids. Deresiewicz ${ }^{6}$ showed the existence of a Rayleigh-type wave that propagates along the free surface of a poroelastic half-space and analyzed the frequency-dependent phase velocity and attenuation.

For a fluid/poroelastic-medium configuration, Rosenbaum $^{5}$ predicted the existence of the pseudo-Rayleigh $(p R)$ and the pseudo-Stoneley $(p S t)$ wave. The latter was explicitly named as such by Feng and Johnson, ${ }^{7,8}$ since a pseudo interface wave has part of its energy leaking into slower bulk modes as it propagates along the interface. Feng and Johnson $^{7}$ also showed the existence of another interface wavemode, the nonleaky true interface wave. It was found that the existence of this wave depends on whether or not the pores are open for pore fluid to flow across the interface.

\footnotetext{
a) Author to whom correspondence should be addressed. Electronic mail: k.n.vandalen@tudelft.nl
}

Feng and Johnson ${ }^{8}$ derived Green's functions (impulse responses) for high-frequency Biot theory that confirmed the existence of the three different waves.

Experimental evidence was found for all three types of interface wavemodes, see, e.g., Refs. 9-11.

Feng and Johnson ${ }^{7}$ argued that other zeroes of the poroelastic Stoneley dispersion equation have no physical significance as pseudo interface modes. The corresponding propagation velocities would be larger than that of shear waves, which is not realistic.

In order to obtain the characteristics of the interface wavemodes, Feng and Johnson ${ }^{7}$ used the zeroes of the nonviscid poroelastic Stoneley dispersion equation in the complex plane to obtain the propagation velocities and attenuations. Gubaidullin et al. ${ }^{12}$ went a step further and analyzed the frequency dependence of the interface wavemodes by incorporating the viscous loss mechanism of Johnson et al. ${ }^{13}$ They also used the zeroes of the dispersion equation to derive the characteristics of the interface waves. The same approach was adopted by Edelman and Wilmanski, ${ }^{14}$ Albers, ${ }^{15}$ and Markov. ${ }^{16}$ In most of the papers, specific restrictions for the involved square roots (i.e., their Riemann sheets) are given.

The generally accepted explanation for a pseudo interface wave is that it originates from a zero that forms a pole singularity on another Riemann sheet than the so-called "principal" sheet. It affects the behavior of the integrand on the principal Riemann sheet by causing a local maximum in the integrand. ${ }^{17}$ In case the pole lies close to the real axis, it might have a contribution to the Green's function.

In a series of publications, Allard et al. ${ }^{11,18,19}$ studied the propagation of interface waves along the boundaries of poroelastic and nonporous elastic media. In the case of an air/ air-saturated poroelastic-solid configuration, they found that 
taking the residue of the pseudo-Rayleigh pole is sufficient to describe the entire pseudo-Rayleigh waveform. ${ }^{19}$ For the water/water-saturated poroelastic-solid configuration, they found that the pseudo-Stoneley pole residue describes the entire waveform of the pseudo-Stoneley wave. ${ }^{11}$ However, for the water/elastic-solid configuration, they found that the pseudo-Rayleigh waveform is strongly affected by the loop integrals along the branch cuts.

In summary, taking just the location of the zeroes of the dispersion equation rather than computing the full transient response is a very fast way to predict the kinematic properties of pseudo interface waves, but the question arises if these predictions are always complete.

Therefore, in this paper we analyze the threedimensional transient wave propagation due to a point force applied at the interface of a fluid and a fluid-saturated poroelastic solid. The aims are as follows.

(1) To investigate if a zero of the poroelastic Stoneley dispersion equation indeed yields the pertinent physical properties of the corresponding pseudo interface wavemode. This is done by quantitative comparison between the residues of specific poles and the full transient response.

(2) To verify if a pseudo interface wave indeed necessarily originates from a pole on a nonprincipal Riemann sheet.

(3) To verify the physical significance of additional zeroes of the poroelastic Stoneley dispersion equation that are not related to pseudo interface waves. ${ }^{7}$

The paper is organized as follows. In Sec. II, we present the model to analyze the fluid/poroelastic-medium configuration. Subsequently, in Sec. III, the derivation of Green's function is summarized. The implementation of the numerical integration is discussed in Sec. IV. We discuss the results in Sec. V. The conclusions are given in Sec. VI.

\section{MODEL}

To study the transient wave propagation in a fluid/ poroelastic-medium configuration, we consider a configuration that consists of a fluid half-space on top of a fluidsaturated poroelastic half-space. A vertical point force $F(t)$ is applied at the interface [see Fig. 1(a); Fig. 1(b) is referred to later]. Both half-spaces are considered to be homogeneous and isotropic. The configuration is similar to the one applied by Gubaidullin et al. ${ }^{12}$ but extended to three dimensions.

The behavior in the lower half-space $\left(x_{3}>0\right)$ is governed by the well-known Biot equations of motion for a fluid-saturated poroelastic solid that were extensively discussed in this journal, see, e.g., Refs. 20 and 21. Following Biot's theory, we assume that for long wavelength disturbances with respect to the characteristic pore scale, average local displacements can be defined for the solid (frame) $\mathbf{u}(\mathbf{x}, t)=\left(u_{1}, u_{2}, u_{3}\right)^{T}$ and the fluid $\mathbf{U}(\mathbf{x}, t)=\left(U_{1}, U_{2}, U_{3}\right)^{T}$. Considering a cube of unit size of bulk material (porosity $\phi$ ), the forces per unit bulk area applied to that part of the cube faces occupied by the solid are denoted by $\tau_{i j}$. They are constituted by both fluid pressure $p_{f}$ and intergranular stresses $\sigma_{i j}$ according to
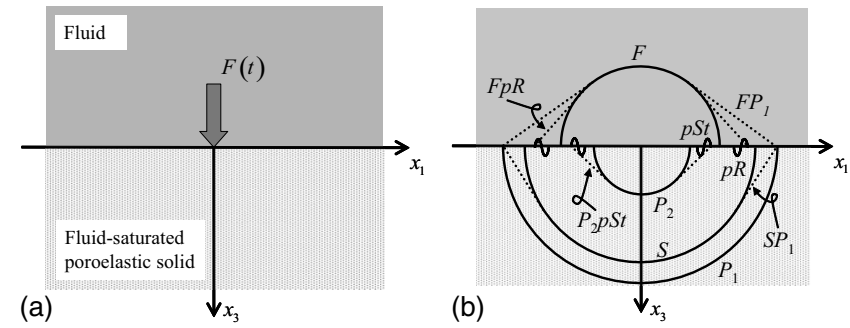

FIG. 1. (a) Point force $F(t)$ applied at the interface of a fluid-saturated poroelastic half-space and a fluid half-space. Both half-spaces are homogeneous and isotropic. (b) Schematic snapshot of the full response with separate arrivals: fast compressional $\left(P_{1}\right)$ wave, slow compressional $\left(P_{2}\right)$ wave, shear $(S)$ wave, fluid $(F)$ wave, pseudo-Rayleigh $(p R)$ wave, and pseudoStoneley $(p S t)$ wave. The double-mode symbols (e.g., $\left.S P_{1}\right)$ indicate lateral waves $(\cdots)$. The first symbol denotes the wavemode of the specific arrival; the second denotes the one from which it is radiated. Here, the $F$-wave velocity is assumed higher than the $P_{2}$-wave velocity. For clarity, we omitted the following arrivals: $F S, P_{2} P_{1}, P_{2} S, P_{2} p R$, and $P_{2} F$.

$$
\tau_{i j}=-\sigma_{i j}-(1-\phi) p_{f} \delta_{i j},
$$

where $\delta_{i j}$ is the Kronecker delta. The total normal tension force per unit bulk area applied to the fluid faces of the unit cube, denoted by $\tau$, is constituted by $p_{f}$ only,

$$
\tau=-\phi p_{f}
$$

Here, $\sigma_{i j}$ and $p_{f}$ are defined positive in compression and, consequently, $\tau_{i j}$ and $\tau$ are positive in tension, see also Ref. 12. In the case of isotropic materials, the stress-strain relations for the solid and the fluid can be written as

$$
\begin{aligned}
\tau_{i j} & =G\left(\partial_{i} u_{j}+\partial_{j} u_{i}\right)+A \partial_{k} u_{k} \delta_{i j}+Q \partial_{k} U_{k} \delta_{i j}, \\
\tau & =Q \partial_{k} u_{k}+R \partial_{k} U_{k},
\end{aligned}
$$

where Einstein's summation convention for repeated indices is applied, and $\partial_{j}=\partial / \partial x_{j} . A, Q$, and $R$ are generalized elastic constants that can be related via Gedanken experiments to porosity, grain bulk modulus $K_{s}$, fluid bulk modulus $K_{f}$, bulk modulus of porous drained frame $K_{b}$, and shear modulus $G$ of both drained frame and total composite. ${ }^{22,23}$ The physical background of Eqs. (3) and (4) is discussed in more detail in Ref. 20.

The equations of motion are found from combination of momentum conservation and the stress-strain relations, Eqs. (3) and (4), and can be written as ${ }^{20,21}$

$$
\begin{aligned}
\rho_{11} \partial_{t}^{2} \mathbf{u}+\rho_{12} \partial_{t}^{2} \mathbf{U}+b * \partial_{t}(\mathbf{u}-\mathbf{U})= & P \nabla \nabla \cdot \mathbf{u}-G \nabla \times \nabla \times \mathbf{u} \\
& +Q \nabla \nabla \cdot \mathbf{U},
\end{aligned}
$$

$\rho_{12} \partial_{t}^{2} \mathbf{u}+\rho_{22} \partial_{t}^{2} \mathbf{U}-b * \partial_{t}(\mathbf{u}-\mathbf{U})=Q \nabla \nabla \cdot \mathbf{u}+R \nabla \nabla \cdot \mathbf{U}$,

where the asterisk denotes convolution, $P=A+2 G$, and the effective densities are defined as

$$
\begin{aligned}
& \rho_{11}=(1-\phi) \rho_{s}-\rho_{12}, \\
& \rho_{22}=\phi \rho_{f}-\rho_{12}, \\
& \rho_{12}=-\left(\alpha_{\infty}-1\right) \phi \rho_{f},
\end{aligned}
$$

where the tortuosity $\alpha_{\infty} \geq 1$, and hence $\rho_{12} \leq 0$. Solid and fluid densities are denoted as $\rho_{s}$ and $\rho_{f}$, respectively. The 
linear time-convolution operator $b$ was formulated in the frequency domain as the viscous correction factor by Johnson et al., ${ }^{13}$ according to

$$
\hat{b}(\omega)=b_{0}\left(1+\frac{1}{2} i M \omega / \omega_{c}\right)^{1 / 2},
$$

where the viscous damping factor $b_{0}=\phi^{2} \eta / k_{0}$. Here, the dynamic fluid viscosity is denoted by $\eta$ and $k_{0}$ represents the zero-frequency Darcy permeability. The shape factor $M$ is usually taken equal to $1 .^{24}$ The rollover frequency, which represents the transition from low-frequency viscositydominated to high-frequency intertia-dominated behavior, is defined as $\omega_{c}=\eta \phi /\left(\alpha_{\infty} \rho_{f} k_{0}\right)$.

The behavior of the upper (fluid) half-space $\left(x_{3}<0\right)$ is governed by the acoustic wave equation

$$
\rho_{F} \partial_{t}^{2} p_{F}=K_{F} \nabla^{2} p_{F}
$$

where $K_{F}$ and $\rho_{F}$ denote the bulk modulus and density of the fluid, respectively, and $p_{F}$ denotes the fluid pressure.

We assume that the behavior at the interface is governed by conventional open-pore conditions, i.e., by continuity of volume flux and fluid pressure, and vanishing intergranular vertical and shear stresses. The force is applied to the solid. The open-pore boundary is a realistic choice to model the fluid/poroelastic-medium interface, ${ }^{1}$ and a limiting case of the situation where a finite surface flow impedance is considered, see e.g., Refs. 12, 25, and 26. It implies that the true interface wave is absent in the response. ${ }^{7,8}$ Hence, in the limit of $x_{3} \rightarrow 0$, the following conditions should be satisfied

$$
\begin{aligned}
& (1-\phi) u_{3}+\phi U_{3}-U_{F, 3}=0, \\
& p_{f}-p_{F}=0, \\
& \sigma_{13}=0, \\
& \sigma_{23}=0, \\
& \sigma_{33}=F(t) \delta\left(x_{1}\right) \delta\left(x_{2}\right),
\end{aligned}
$$

where $\delta(\cdots)$ denotes the Dirac delta function, and $U_{F, 3}$ denotes the vertical particle displacement in the upper halfspace. The fact that the intergranular stress $\sigma_{33}$ is zero does not imply that the total solid stress $\tau_{33}$ vanishes, see Eq. (1).

The medium is considered to be at rest at $t \leq 0$. At infinite distance from the source, the motions are bounded.

\section{GREEN'S FUNCTIONS}

In this section, we summarize the derivation of the Green's functions (impulse responses) as described by the solution to the set of governing equations, Eqs. (5), (6), and (9)-(14). The main part of the derivation is given in Appendices $\mathrm{A}$ and $\mathrm{B}$ and we refer to them where necessary.

In order to analyze the response in the plane-wave domain, the Fourier transform is applied over time according to

$$
\hat{\mathbf{u}}(\mathbf{x}, \omega)=\int_{-\infty}^{\infty} \mathbf{u}(\mathbf{x}, t) \exp (-i \omega t) d t
$$

where $\omega$ denotes radial frequency. It is assumed that $\mathbf{u}(\mathbf{x}, t)$ is real valued and hence, it is sufficient to consider $\omega \geq 0$. Fol- lowing Aki and Richards, ${ }^{4}$ the Fourier transform over horizontal spatial coordinates can be defined as

$$
\widetilde{\mathbf{u}}\left(\mathbf{p}, x_{3}, \omega\right)=\int_{-\infty}^{\infty} \int_{-\infty}^{\infty} \hat{\mathbf{u}}(\mathbf{x}, \omega) \exp (i \omega \mathbf{p} \cdot \mathbf{r}) d x_{1} d x_{2},
$$

where $\mathbf{p}=\left(p_{1}, p_{2}\right)^{T}$ is the horizontal slowness vector and $\mathbf{r}$ $=\left(x_{1}, x_{2}\right)^{T}$ is the horizontal space vector. The transforms are applied similarly to the other field quantities. The hat refers to the $(\mathbf{x}, \omega)$-domain and the tilde to the $\left(\mathbf{p}, x_{3}, \omega\right)$-domain.

The response in the $\left(\mathbf{p}, x_{3}, \omega\right)$-domain is described by the physical quantities $\tilde{u}_{i}$ and $\tilde{p}_{f}$ in the lower half-space, collected in the vector $\widetilde{\mathbf{w}}=\left(\tilde{u}_{1}, \widetilde{u}_{2}, \tilde{u}_{3},-\phi \widetilde{p}_{f}\right)^{T}$, and by $\widetilde{p}_{F}$ in the upper half-space, see Eqs. (A1), (A4), and (A6). The expressions for the response are obtained using Helmholtz decomposition of the equations of motion and substitution of the general solutions into the boundary conditions. This gives a set of equations that is solved analytically (see Appendix A).

The response can be written in terms of Green's functions according to

$$
\begin{gathered}
\widetilde{\mathbf{w}}=\widetilde{\mathbf{g}}^{+} \hat{F}=\frac{\widetilde{\mathbf{n}}^{+}}{\Delta_{S t}} \hat{F}, \\
\tilde{p}_{F}=\widetilde{g}^{-} \hat{F}=\frac{\widetilde{n}^{-}}{\Delta_{S t}} \hat{F},
\end{gathered}
$$

where $\widetilde{\mathbf{g}}^{+}$and $\widetilde{g}^{-}$are the Green's functions in the lower and upper media, respectively, $\widetilde{\mathbf{n}}^{+}$and $\tilde{n}^{-}$are the corresponding numerators, and $\hat{F}$ is the Fourier transform of the force signature. From Eqs. (A1), (A4), and (A6), it follows that $\widetilde{\mathbf{g}}^{+}$ consists of a superposition of all possible body modes: the fast $\left(P_{1}\right)$ and slow $\left(P_{2}\right)$ compressional waves, and the vertically polarized shear $(S V)$ wave. The horizontally polarized shear $(S H)$ mode is not excited by the vertical force. The Green's function $\widetilde{g}^{-}$only contains the fluid $(F)$ compressional mode. Both Green's functions have the "poroelastic Stoneley-wave denominator" $\Delta_{S t}=\Delta_{S t}(p, \omega)$ that is associated with interface waves along the fluid/poroelastic-medium interface, which is very similar to the "Scholte-wave denominator" for a fluid/elastic-solid interface. ${ }^{27}$ Here, $p=\left(p_{1}^{2}\right.$ $\left.+p_{2}^{2}\right)^{1 / 2}$ denotes the magnitude of the horizontal slowness.

The body-wave slownesses $s_{j}, j=\left\{P_{1}, P_{2}, F, S\right\}$, are defined in Appendix A (Table III). The corresponding vertical slownesses are defined as $q_{j}=\left(s_{j}^{2}-p^{2}\right)^{1 / 2}$, where $\operatorname{Im}\left(q_{j}\right) \leq 0$ due to Sommerfeld's radiation condition.

To find the Green's functions in the $(\mathbf{x}, \omega)$-domain, the inverse Fourier transform is applied according to

$$
\hat{\mathbf{g}}^{+}=\frac{\omega^{2}}{(2 \pi)^{2}} \int_{-\infty}^{\infty} \int_{-\infty}^{\infty} \frac{\widetilde{\mathbf{n}}^{+}\left(\mathbf{p}, x_{3}, \omega\right)}{\Delta_{S t}} \exp (-i \omega \mathbf{p} \cdot \mathbf{r}) d p_{1} d p_{2}
$$

where $\omega \geq 0$. We only show the derivation of $\hat{\mathbf{g}}^{+}$, but the expressions for $\hat{g}^{-}$are obtained by simply replacing $\widetilde{\mathbf{n}}^{+}$by $\tilde{n}^{-}$. When cylindrical coordinates are introduced, Eq. (19) can be written as 


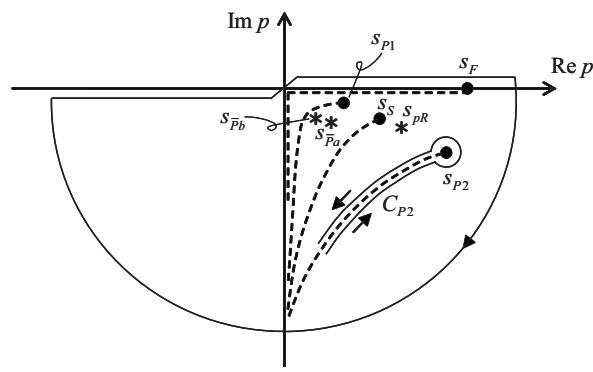

FIG. 2. Complex $p$-plane with $(--)$ branch cuts, $(\bullet)$ branch points $s_{j}, j$ $=\left\{P_{1}, P_{2}, F, S\right\}$, and $(*)$ poles $s_{p R}$ (pseudo-Rayleigh), $s_{\bar{P} a}$ and $s_{\bar{P} b}$ (additional), for the calculation of the Green's functions for Bentheimer/air configuration 2 (see Table I). The branch points are formed by the body-wave slownesses specified in Appendix A (Table III). The hyperbolic branch cuts are described by $\operatorname{Im}\left(q_{j}\right)=0$. Poles are zeroes of the poroelastic Stoneley denominator, see Eq. (21). Only part of the closed integration contour (-) is displayed: real axis, arc in lower half-plane, and loop $C_{P 2}$ along the $q_{P 2}$-branch cut. The direction of integration is indicated.

$$
\hat{\mathbf{g}}^{+}=\frac{\omega^{2}}{4 \pi} \int_{-\infty}^{\infty} \frac{\widetilde{\mathbf{n}}^{+}\left(p, \partial_{\alpha}, x_{3}, \omega\right)}{\Delta_{S t}} H_{0}^{(2)}(\omega p r) p d p
$$

where $r=\left(x_{1}^{2}+x_{2}^{2}\right)^{1 / 2}$ and in which the horizontal derivatives $\partial_{\alpha}, \alpha=\{1,2\}$, are applied to the Hankel function $H_{0}^{(2)}(\cdots)$, see Eqs. (B3) and (B4) (Appendix B).

Now we change the real-axis integral into a contour integral in the complex $p$-plane. The idea is that by integration in the complex plane, contributions from loop integrals and from pole residues can be distinguished. We choose branch cuts along the hyperbolic lines ${ }^{3} \operatorname{Im}\left(q_{j}\right)=0$. In this way $\operatorname{Im}\left(q_{j}\right) \leq 0 \quad \forall p$, which ensures the decay of the exponential terms $\exp \left(\mp i \omega q_{j} x_{3}\right)$ for large $p$ [see Eqs. (A1) and (A4)]. The branch cuts depart from the branch points associated with the body-wave slownesses $s_{j}$, as shown in Fig. 2. The $q_{F}$-branch cut reduces to the imaginary axis and part of the real axis since the slowness of the fluid wave $\left(s_{F}\right)$ is real.

The current branch cuts are referred to as the "fundamental" branch cuts. ${ }^{28}$ The corresponding Riemann sheet is referred to as the principal Riemann sheet ${ }^{17}$ or the "physical" Riemann sheet. ${ }^{4}$

In Fig. 2 the closed contour is also displayed. It is formed by the entire real axis, the loops along the branch cuts and around the branch points, and an arc of infinite radius in the lower half-plane. For $\operatorname{Re}(p) \leq 0$, the horizontal part of the contour lies just below the axis due to the presence of a branch cut of the Hankel function at the negative real axis. ${ }^{29}$

Applying Cauchy's residue theorem, ${ }^{30}$ we obtain

$$
\begin{aligned}
& \hat{\mathbf{g}}^{+}=\int_{-\infty}^{\infty} \tilde{\mathbf{f}}^{+} d p=-2 \pi i \sum_{m} \operatorname{Res}_{p=s_{m}} \tilde{\mathbf{f}}^{+}-\sum_{j} \int_{C_{j}} \tilde{\mathbf{f}}^{+} d p, \\
& \tilde{\mathbf{f}}^{+}=\frac{\omega^{2}}{4 \pi} \frac{\widetilde{\mathbf{n}}^{+}\left(p, \partial_{\alpha}, x_{3}, \omega\right)}{\Delta_{S t}} H_{0}^{(2)}(\omega p r) p,
\end{aligned}
$$

where every $s_{m}$ denotes a first-order pole of the integrand inside the integration contour and every $C_{j}$ denotes a loop along the specific branch cut. In Eq. (21), the contribution of the arc vanishes because of Jordan's lemma. ${ }^{31}$ The contributions around the branch points are also zero.
The poles $s_{m}$ result from zeroes of the poroelastic Stoneley dispersion equation $\left(\Delta_{S t}=0\right)$ on the principal Riemann sheet. The number of poles $N$ present inside contour $C$ is determined by applying the principle of the argument to the Stoneley equation ${ }^{30}$

$$
N=\frac{1}{2 \pi i} \oint_{C} \frac{\partial_{p} \Delta_{S t}}{\Delta_{S t}} d p
$$

The residue of the integrand at a first-order pole is given as

$$
\operatorname{Res}_{p=s_{m}} \tilde{\mathbf{f}}^{+}=\left[\frac{\omega^{2}}{4 \pi} \frac{\widetilde{\mathbf{n}}^{+}\left(p, \partial_{\alpha}, x_{3}, \omega\right)}{\partial_{p} \Delta_{S t}} H_{0}^{(2)}(\omega p r) p\right]_{p=s_{m}} .
$$

\section{NUMERICAL IMPLEMENTATION}

To perform the integration along the hyperbolic branch cuts, we choose $p_{i}=\operatorname{Im}(p)$ as the variable of integration according to

$$
\int_{C_{j}} \widetilde{\mathbf{f}}^{+} \frac{\partial p}{\partial p_{i}} d p_{i}
$$

where

$$
p=\frac{\operatorname{Re}\left(s_{j}\right) \operatorname{Im}\left(s_{j}\right)}{p_{i}}+i p_{i}, \quad \frac{\partial p}{\partial p_{i}}=-\frac{\operatorname{Re}\left(s_{j}\right) \operatorname{Im}\left(s_{j}\right)}{p_{i}^{2}}+i .
$$

For the $q_{F}$-branch cut, the integration path is the imaginary axis and part of the real axis, which follows from Eq. (25) for vanishing imaginary part of the slowness $\operatorname{Im}\left(s_{F}\right) \uparrow 0$ (Fig. 2). Along the cut of $q_{j}$, at the left side $\operatorname{Re}\left(q_{j}\right)>0$ and at the right side $\operatorname{Re}\left(q_{j}\right)<0$. At the specific cut $\operatorname{Im}\left(q_{j}\right)=0$ and everywhere else $\operatorname{Im}\left(q_{j}\right)<0$.

The numerical integration is performed using an adaptive eight-point Legendre-Gauss algorithm, ${ }^{29,32}$ which can handle integrable singularities such as branch points.

For the numerical implementation of the principle of the argument, we apply Eq. (22) separately for the areas between the various parts of the integration contour (branch cuts, real axis, and arc, see Fig. 2) to find out where the poles can be expected. Subsequently, the pole locations are found numerically by minimizing the left-hand side of equation $\left|\Delta_{S t}\right|=0$. Since it contains local minima and branch-cut discontinuities, it is important to choose a proper starting value. This requires some manual iteration. The accuracy, as expressed by $\left|\Delta_{S t}\left(p=s_{m}\right)\right| /\left|\Delta_{S t}(p=0)\right|$, is typically $O\left(10^{-10}\right)$. Here $s_{m}$ denotes the numerical value of the pole location.

\section{NUMERICAL RESULTS AND DISCUSSION}

In this section, we investigate the transient responses for four different fluid/poroelastic-medium configurations (see Table I). In the first three configurations, water-saturated Bentheimer sandstone (see Table II) is used as porous medium. The upper half-space is subsequently filled with water, air, or a light fluid. In the fourth configuration, which is the one of Feng and Johnson, ${ }^{7,8}$ the porous medium is formed by water-saturated fused glass beads, while the upper half-space is filled with water.

For every configuration, we will show the vertical component of particle velocity $v_{3}$ and the fluid pressure $p_{f}$ for an 
TABLE I. Various configurations for which the transient response is calculated. The type of sandstone is Bentheimer. For fused glass beads, the bulk modulus of the drained matrix is chosen as $K_{b}=10 \mathrm{GPa}$ and the permeability is chosen as $k_{0}=10^{-11} \mathrm{~m}^{2}$. The upper half-space is filled with either water $\left(K_{F}=K_{f}, \rho_{F}=\rho_{f}\right)$, or air $\left(K_{F}=1.42 \times 10^{2} \mathrm{kPa}, \rho_{F}=1.25 \mathrm{~kg} \mathrm{~m}^{-3}\right)$, or a light fluid $\left(K_{F}=K_{f} / 10, \rho_{F}=\rho_{f} / 8\right)$. For every configuration, the poles present on the principal Riemann sheet are indicated: pseudo-Stoneley $(p S t)$, pseudo-Rayleigh $(p R)$, and two additional $\left(\bar{P}_{a}, \bar{P}_{b}\right)$ poles.

\begin{tabular}{ccccc}
\hline \hline & Porous solid & Saturating fluid & Upper half-space & Poles \\
\hline 1 & Sandstone & Water & Water & $p S t$ \\
2 & Sandstone & Water & Air & $\bar{P}_{a}, \bar{P}_{b}{ }^{\mathrm{a}}{ }^{a} p R$ \\
3 & Sandstone & Water & Light fluid & $p R, p S t^{\mathrm{a}}$ \\
4 & Fused glass beads & Water & Water & $p S t^{\mathrm{a}}$ \\
\hline \hline
\end{tabular}

${ }^{\mathrm{a}}$ Its residue is not shown.

observation point at the interface $x_{3}=0$ at offset $r=x_{1}$ $=0.1 \mathrm{~m}$. Fluid pressure is related to dilatation only [see Eq. (4)] and hence, $v_{3}$ and $p_{f}$ contain different information. Also, the comparison between the full response and a pole residue can be different in $v_{3}$ and $p_{f}$, as will be shown.

The point force has Ricker signature, ${ }^{33}$

$$
F(t)=F_{\max }\left(\frac{1}{2} \omega_{0}^{2} \vec{t}^{2}-1\right) \exp \left(-\frac{1}{4} \omega_{0}^{2} \vec{t}^{2}\right)
$$

where $\bar{t}=t-t_{s}, \omega_{0}=2 \pi f_{0}$, and center frequency $f_{0}=500 \mathrm{kHz}$ (see Fig. 3). The magnitude $F_{\max }=1 \mathrm{~N}$ and time shift $t_{s}$ $=5 \mu \mathrm{s}$. We perform the integration for the frequency range $0<f \leq 2 \mathrm{MHz}$. The full response is obtained by multiplication of the spectra of the Green's functions and the source [see Eqs. (17) and (18)], and using a standard fast Fourier transform algorithm.

\section{A. Residue contribution vs full response}

First, we address the relation between a pole and a pseudo interface wave, as raised in point (1) in the Introduction (Sec. I). For configurations 1-3, the full transient responses and separate pole residues [see Eq. (21)] are displayed in Figs. 3-5. We identified the different arrivals in the full responses using the propagation velocities as obtained from the modal slownesses. Head waves are identified geometrically using the pertaining modal velocities and are indicated with double-mode symbols [e.g., $S P_{1}$ : the shear $(S)$

TABLE II. Material parameters as used for water-saturated Bentheimer sandstone (Ref. 34). The bulk modulus of the matrix $K_{b}$ is found according to $K_{b}=K_{p}-\frac{4}{3} G$.

\begin{tabular}{lc}
\hline \hline Solid (frame) density $\rho_{s}$ & $2630 \mathrm{~kg} \mathrm{~m}^{-3}$ \\
Fluid density $\rho_{f}$ & $1000 \mathrm{~kg} \mathrm{~m}^{-3}$ \\
Tortuosity $\alpha_{\infty}$ & 2.4 \\
Porosity $\phi$ & 0.23 \\
Permeability $k_{0}$ & $3.7 \mu \mathrm{m}^{2}$ \\
Dynamic fluid viscosity $\eta$ & $0.001 \mathrm{~Pa} \mathrm{~s}$ \\
Shear modulus $G$ & $6.8 \mathrm{GPa}$ \\
Constrained modulus $K_{p}$ & $14 \mathrm{GPa}$ \\
Grain bulk modulus $K_{s}$ & $36.5 \mathrm{GPa}$ \\
Fluid bulk modulus $K_{f}$ & $2.22 \mathrm{GPa}$ \\
\hline \hline
\end{tabular}

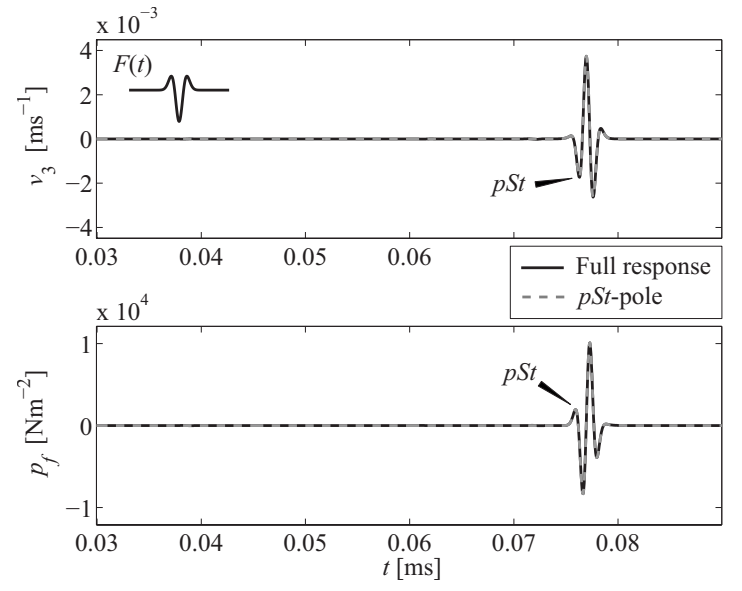

FIG. 3. Full response and $p S t$-pole residue at $x_{2}=x_{3}=0$ and offset $x_{1}$ $=0.10 \mathrm{~m}$ for Bentheimer/water configuration 1 . The $p S t$-pole residue coincides with the $p S t$-waveform in the full response. Other wavemodes are too weak to be observed in this figure. The source signature $F(t)$ is also displayed.

wave radiated by the fast $\left(P_{1}\right)$ compressional wave]. For the sake of clarity, a schematic snapshot of the full response with the different arrivals is shown in Fig. 1(b).

We first note that the $P_{1}$-wave is present quite strongly in $v_{3}$ although this component is perpendicular to the direction of propagation of this longitudinal wave (Figs. 4 and 5). This is due to the contraction in vertical direction that can easily take place at the air/sandstone or light-fluid/sandstone interface. Remarkably, there is an arrival present in $p_{f}$ at the $S$-wave arrival time (Figs. 4 and 5). This is not an $S$-wavefront but radiated slow compressional $\left(P_{2}\right)$ and fluid $(F)$ head waves, see Fig. 1(b).

Now, we focus on the comparison of interface waves in the full responses and corresponding pole residues. The pole(s) that are present on the principal Riemann sheet contributing a residue are given in Table I, for each configuration separately. We found the pseudo-Stoneley $(p S t)$, the pseudoRayleigh $(p R)$, and two additional $\left(\bar{P}_{a}, \bar{P}_{b}\right)$ poles. The latter ones are discussed in Sec. V C.

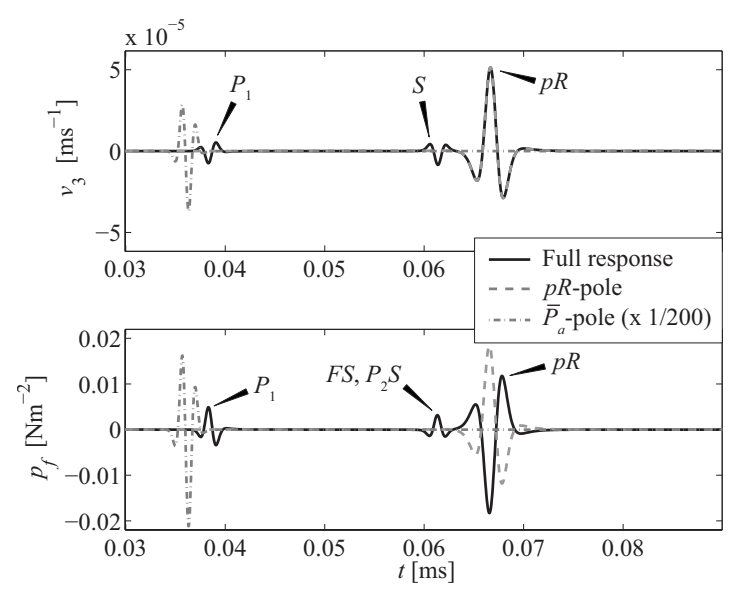

FIG. 4. Full response and residues of $p R$-pole and $\bar{P}_{a}$-pole at $x_{2}=x_{3}=0$ and offset $x_{1}=0.10 \mathrm{~m}$ for Bentheimer/air configuration 2. The $\bar{P}_{a}$-pole residue has been scaled down by a factor 200 to make it entirely visible. The $p R$-pole residue coincides with the $p R$-waveform in the full response of $v_{3}$. The double-mode symbols are explained in Fig. 1(b). 


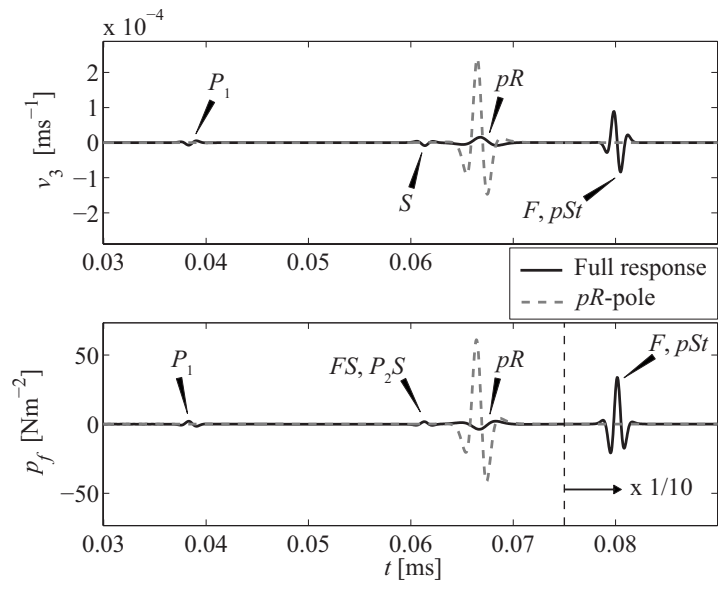

FIG. 5. Full response and $p R$-pole residue at $x_{2}=x_{3}=0$ and offset $x_{1}$ $=0.10 \mathrm{~m}$ for Bentheimer/light-fluid configuration 3 . From the dashed vertical line onwards, the response $p_{f}$ has been scaled down by a factor 10 to make it entirely visible. The double-mode symbols are explained in Fig. 1(b).

For configuration 1 (water as upper fluid), only the $p S t$-pole is found on the principal Riemann sheet. From Fig. 3 , we observe that its residue yields the entire $p S t$-waveform. For configuration 2 (air as upper fluid), the $p R$-pole is found on the principal Riemann sheet. From Fig. 4, it is observed that its residue coincides with the $p R$-waveform in the full response of $v_{3}$ (actually, the difference is nonzero but too small to be observed). However, it does not coincide with that in the full response of $p_{f}$. Its contribution is opposite, which means that the loop integrals along the branch cuts also contribute to the pseudo interface waveform. This was also found by Allard et al. ${ }^{11}$ It implies that part of the pertinent physical properties of the pseudo interface wave is captured by the loop integrals.

This is more pronounced for configuration 3 (light upper fluid), as shown in Fig. 5, in which both the $p R$-pole and the $p S t$-pole are found on the principal Riemann sheet. In both components $\left(v_{3}\right.$ and $\left.p_{f}\right)$, the $p R$-pole residue does not coin-

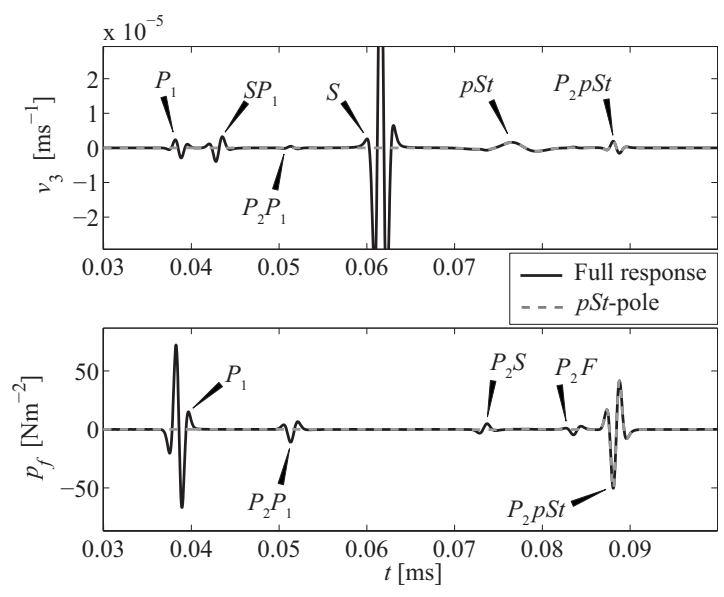

FIG. 6. Full response and $p S t$-pole residue at $x_{2}=0, x_{3}=0.01 \mathrm{~m}$, and offset $x_{1}=0.10 \mathrm{~m}$ for Bentheimer/water configuration 1. The $p S t$-pole residue coincides with the $p S t$ - and $P_{2} p S t$-waveforms in the full response. The doublemode symbols are explained in Fig. 1(b).

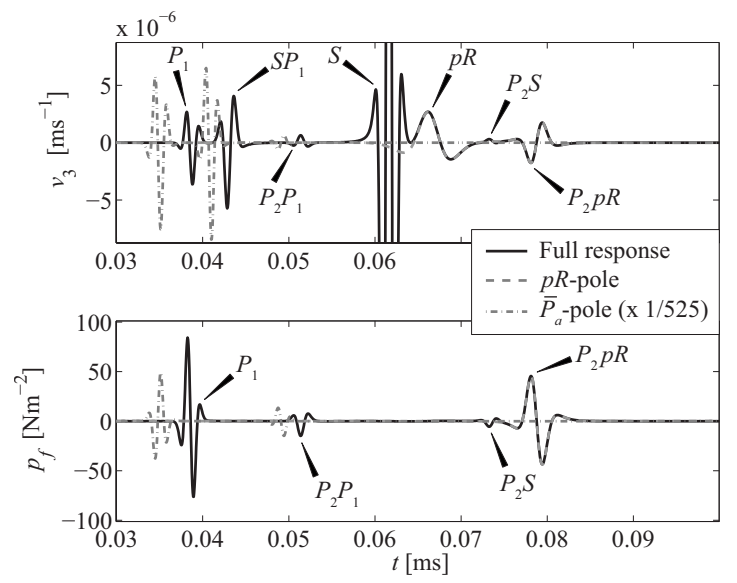

FIG. 7. Full response and residues of $p R$-pole and $\bar{P}_{a}$-pole at $x_{2}=0, x_{3}$ $=0.01 \mathrm{~m}$, and offset $x_{1}=0.10 \mathrm{~m}$ for Bentheimer/air configuration 2 . The $p R$-pole residue coincides with the $p R$ - and the $P_{2} p R$-waveforms in the full response. The $\bar{P}_{a}$-pole residue has been scaled down by a factor 525 to make it entirely visible. The double-mode symbols are explained in Fig. 1(b).

cide with the $p R$-waveform in the full response. The $p S t$-pole residue is not displayed separately because the pSt-wave strongly interferes with the $F$-wave.

To investigate how the residues and the interface waveforms in the full responses compare for an observation point that lies off the interface, we calculated the responses for configurations 1 to 3 at $x_{3}=0.01 \mathrm{~m}$ and offset $r=x_{1}=0.1 \mathrm{~m}$. The corresponding results are displayed in Figs. 6-8. Compared to the previous responses at $x_{3}=0$, various head waves can now be distinguished as separate arrivals, generated by the body wavefronts that propagate along the interface, $\mathrm{cf}$. Fig. 1(b). From Figs. 6-8, we also observe that the residues now yield two waveforms in the full responses. The first one ( $p R$ or $p S t)$ is the waveform of the specific interface wave itself, while the second $\left(P_{2} p R\right.$ or $\left.P_{2} p S t\right)$ corresponds to the $P_{2}$-mode that is radiated by the propagating pseudo interface wave. For configurations 1 (water as upper fluid, Fig. 6) and 2 (air as upper fluid, Fig. 7), it is observed that both waveforms are now captured entirely by the residue of the corresponding pole. For configuration 3 (light upper fluid, Fig. 8), this is not the case, as for $x_{3}=0$.

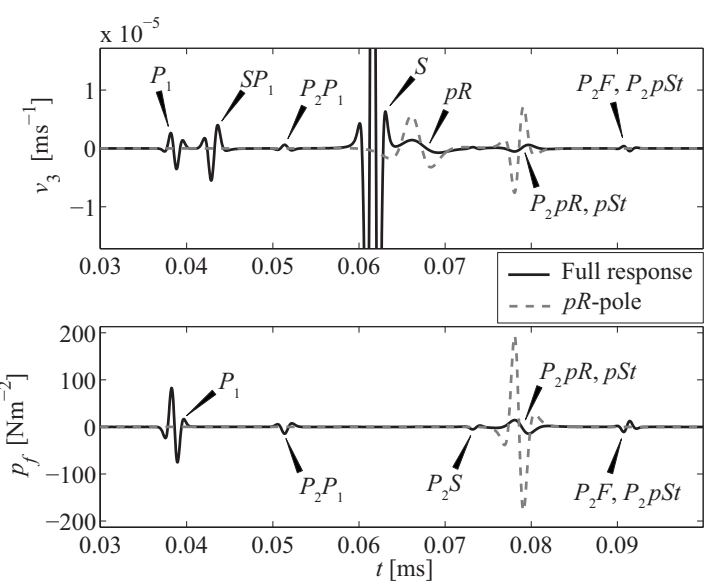

FIG. 8. Full response and $p R$-pole residue at $x_{2}=0, x_{3}=0.01 \mathrm{~m}$, and offset $x_{1}=0.10 \mathrm{~m}$ for Bentheimer/light-fluid configuration 3. The double-mode symbols are explained in Fig. 1(b). 


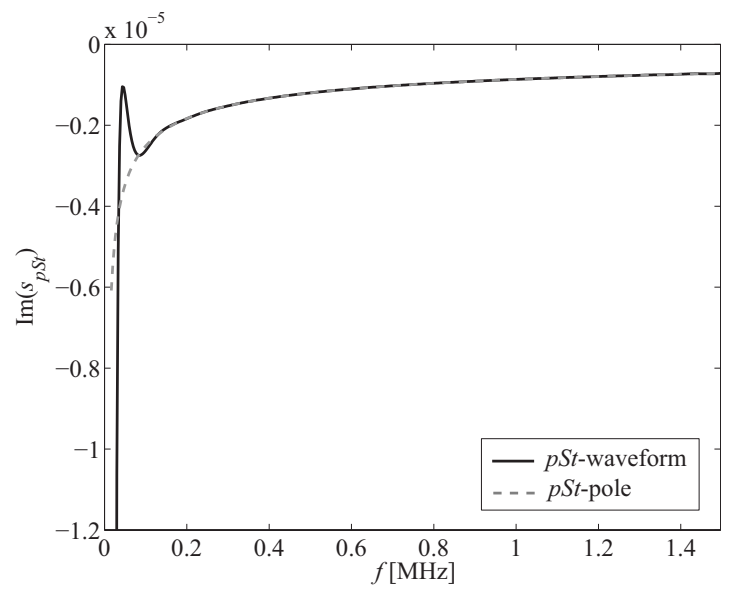

FIG. 9. True attenuation, defined as $\operatorname{Im}\left(s_{p S t}^{\mathrm{tr}}\right)$, retrieved from windowed $p S t$-waveforms for Bentheimer/water configuration 1, at $x_{2}=x_{3}=0$ and fromoffsets $x_{1}=0.24-0.26 \mathrm{~m}$. The attenuation $\operatorname{Im}\left(s_{p S t}\right)$ obtained from the corresponding pole residue is also displayed.

In addition to the observations on responses with entire waveforms, we give attention to the characteristics of a pseudo interface wave. With regard to the propagation velocity, we observe that it is predicted properly by the residue of the corresponding pole for all presented numerical results. Concerning the attenuation, it was proposed by van der Hijden $^{17}$ to quantify the true attenuation of a pseudo interface wave based on the full transient response. This is also done by Rosenbaum, ${ }^{5}$ but he only showed the decay of the total waveform, which would result in one value for the attenuation. This is quite restrictive and therefore we use the following method to retrieve the frequency-dependent attenuation from a windowed pseudo interface waveform in the full response. Here, attenuation is defined by $\operatorname{Im}\left(s_{m}^{\mathrm{tr}}\right)$, where $s_{m}^{\mathrm{tr}}$ represents the true wave slowness and $m$ $=\{p R, p S t\}$. As a starting point, we consider the pseudo interface wave in the far field where it does not interfere with other wavemodes, and we assume that it is described by

$$
\hat{v}_{m, 3}(r) \propto r^{-1 / 2} \exp \left(-i \omega s_{m}^{\mathrm{tr}} r\right),
$$

which is found from the asymptotic behavior of the Hankel function. ${ }^{29}$ The imaginary part of the wave slowness can be retrieved by comparing the amplitude spectra of the windowed waveform $\left|\hat{v}_{m, 3}(r)\right|$ at two different observation points $r=r_{a}$ and $r=r_{b}$, according to

$$
\operatorname{Im}\left(s_{m}^{\mathrm{tr}}(f)\right)=\frac{1}{2 \pi f\left(r_{b}-r_{a}\right)} \ln \left(\frac{r_{b}^{1 / 2}\left|\hat{v}_{m, 3}\left(r_{b}\right)\right|}{r_{a}^{1 / 2}\left|\hat{v}_{m, 3}\left(r_{a}\right)\right|}\right) .
$$

For configurations 1 (water as upper fluid) and 3 (light upper fluid), the attenuations are displayed in Figs. 9 and 10, respectively, together with the corresponding predictions obtained from the poles $p=s_{m}$. The limited frequency range is due to the limited bandwidth of the retrieved spectra. For configuration 1 (water as upper fluid), we observe that the attenuation is described very well by the $p S t$-pole, except for the low frequencies where the far-field approximation of the Hankel function in Eq. (27) is not valid. For configuration 3 (light upper fluid), however, the true attenuation of the $p R$-wave is much greater than the value obtained from the

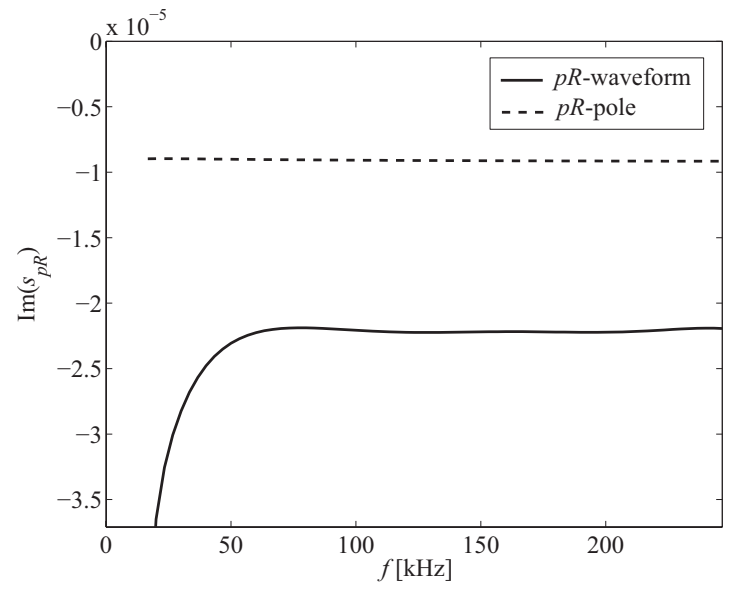

FIG. 10. True attenuation, defined as $\operatorname{Im}\left(s_{p R}^{\mathrm{tr}}\right)$, retrieved from windowed $p R$-waveforms for Bentheimer/light-fluid configuration 3, at $x_{2}=x_{3}=0$ and from offsets $x_{1}=0.24-0.26 \mathrm{~m}$. The attenuation $\operatorname{Im}\left(s_{p R}\right)$ obtained from the corresponding pole is also displayed.

$p R$-pole residue. Obviously, the loop integrals along the branch cuts cannot only affect the waveform but also the spatial decay of a pseudo interface wave.

Sometimes, a residue of a pole is (implicitly) considered to represent the corresponding interface-wave part of the spectrum of the Green's function (see e.g., Refs. 7, 12, and 14-16), while the loop integrals are considered to constitute the part related to body waves and head waves (if present). This can be true but we emphasize that the choice of branch cuts is not unique. Therefore, the integration can be performed on another physically allowed Riemann sheet, i.e., a Riemann sheet that also meets the requirement of $\operatorname{Im}\left(q_{j}\right)$ $\leq 0$ for real $p,{ }^{2,4}$ which is the original path of integration [see Eq. (20)]. This was done by Allard et al. ${ }^{11}$ and Tsang, ${ }^{35}$ and clarified by Harris and Achenbach. ${ }^{36}$ Then, the construction of the $(\mathbf{x}, \omega)$-domain Green's function is different as other poles have to be taken into account and different loop integrals are to be evaluated. Therefore, it might very well be that (part of) the pertinent physical properties of a true or pseudo interface wave are captured by the integrals along the closed contour, rather than by the residue of a specific pole (alone).

From the current observations, we conclude that a residue of a pole on the principal Riemann sheet does not necessarily yield all the pertinent physical properties of the corresponding pseudo interface wave.

\section{B. Presence of $p R$-pole and $p S t$-pole on Riemann sheets}

Now we address the issue concerning the origin of a pseudo interface wave, as raised in point (2) of the Introduction (Sec. I). In the computations in Sec. V A, we already found that a pole related to a pseudo interface wave can be located on the principal Riemann sheet and, obviously, contribute a residue to the full response (see Table I). This contradicts the conventional explanation that a pseudo interface wave originates from a pole on a different Riemann sheet and is accounted for only by the loop integrals along branch cuts by causing a local maximum in the integrand. Allard et 

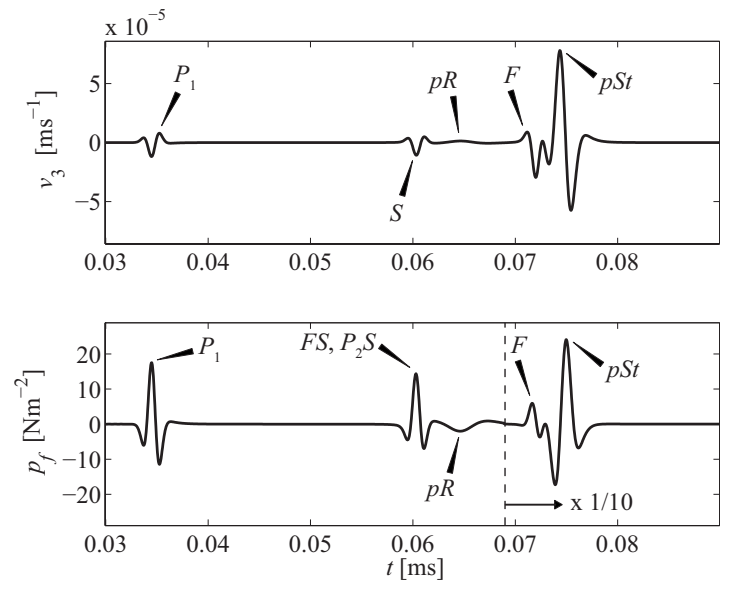

FIG. 11. Full response at $x_{2}=x_{3}=0$ and offset $x_{1}=0.10 \mathrm{~m}$ for fused glassbeads/water configuration 4 . From the dashed vertical line onwards, the response $p_{f}$ has been scaled down by a factor 10 to make it entirely visible. The double-mode symbols are explained in Fig. 1(b).

$a l^{11}$ already found this contradiction, but they did not refer to this as such because their concern was to determine whether or not a pole is related to a separate arrival in the full response.

Surprisingly, in case of fused glass beads saturated with water and covered with water (configuration 4, Fig. 11) the $p S t$-pole is present on the principal Riemann sheet only for a limited frequency range. In Fig. 12, the position of the pole in the complex plane is given, as expressed by $\operatorname{Im}\left(s_{p S t}\right)$. Also the position of the $q_{P 2}$-branch cut is displayed, as expressed by its imaginary part at $\operatorname{Re}(p)=\operatorname{Re}\left(s_{p S t}\right)$. As frequency increases, the $p S t$-pole moves toward the branch cut and as soon as it reaches the cut, it vanishes from the sheet. The pole is not present on the principal sheet for $310 \mathrm{kHz}<f$ $\leq 2 \mathrm{MHz}$. Therefore, the residue of the pSt-pole is not shown in Fig. 11. For the material properties used by Gubaidullin et al., ${ }^{12}$ exactly the same situation occurs, although the transition takes place at a different frequency. Obviously, the presence of a pole on a certain Riemann sheet

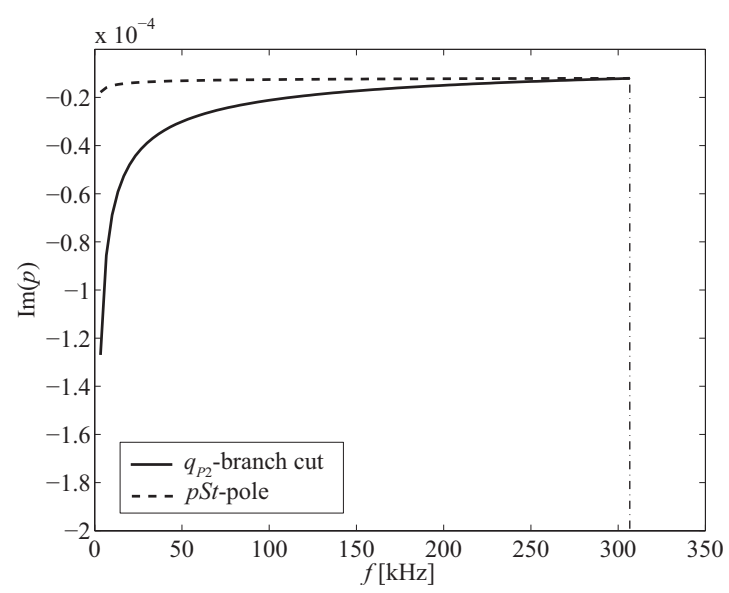

FIG. 12. The location of the $p S t$-pole in the complex $p$-plane, as expressed by $\operatorname{Im}(p)=\operatorname{Im}\left(s_{p S t}\right)$, with respect to the location of the $q_{P 2}$-branch cut at $p$ $=\operatorname{Re}\left(s_{p S t}\right)$, as expressed by $\operatorname{Im}(p)=\operatorname{Re}\left(s_{P 2}\right) \operatorname{Im}\left(s_{P 2}\right) / \operatorname{Re}\left(s_{p S t}\right)$, for fused glass beads/water configuration 4 . The (-.) line indicates the frequency at the intersection.

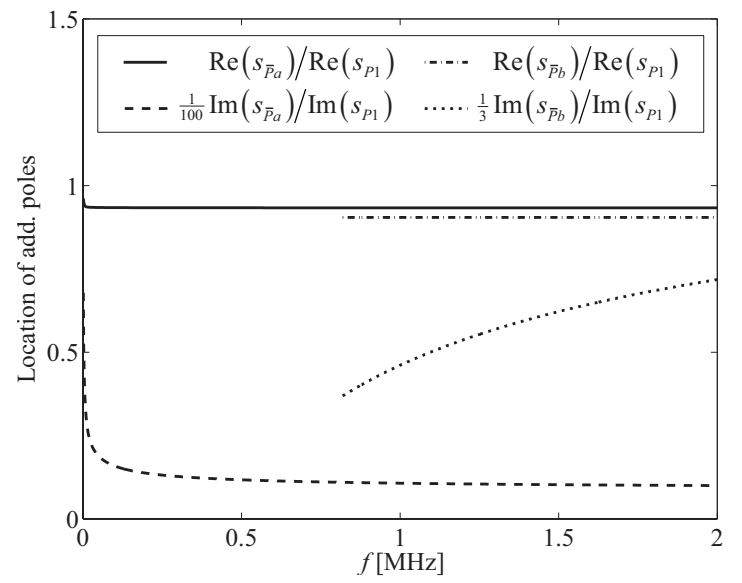

FIG. 13. The location of the additional poles $p=s_{P a}$ and $p=s_{P b}$ in the complex $p$-plane with respect to the $P_{1}$-wave slowness $s_{P 1}$. The $\bar{P}_{b}$-pole is only present on the principal Riemann sheet for limited frequency range $818.75 \mathrm{kHz} \leq f \leq 2 \mathrm{MHz}$.

is not only a matter of the contrast in material parameters of the half-spaces ${ }^{37}$ but can also depend on frequency in case of viscous poroelastic media.

The behavior of the $p S t$-pole illustrates both the nonconventional and the conventional explanation about the origin of a pseudo interface wave. The pole does contribute a residue over a certain frequency range and not outside that specific range. For the $p R$-wave present in the full response of configuration 4 (Fig. 11), only the conventional explanation holds as the $p R$-pole is not found on the principal Riemann sheet and the entire waveform is captured by the loop integrals.

\section{Physical significance of additional poles}

Finally, we give attention to the physical significance of two additional zeroes of the poroelastic Stoneley dispersion equation $\left(\Delta_{S t}=0\right)$ as raised in point (3) of the Introduction (Sec. I). In configuration 2 (air as upper fluid), these zeroes show up as poles on the principal Riemann sheet at $p=s_{\bar{P} a}$ and $p=s_{P}$. They are located to the left of the fast compressional-wave slowness $\left[\operatorname{Re}\left(s_{\bar{P} a}, \bar{P} b\right)<\operatorname{Re}\left(s_{P 1}\right)\right]$ close to the $q_{P 1}$-branch cut (see Fig. $2 ; p=s_{P a}$ a signifies the pole that lies the closest to $\left.p=s_{P 1}\right)$. The additional $\left(\bar{P}_{a}, \bar{P}_{b}\right)$ poles are comparable with the so-called $\bar{P}$-poles that occur in nonporous elastic solids with an interface, as described by Gilbert and Laster ${ }^{38}$ and Aki and Richards. ${ }^{4}$ The scaled real and imaginary parts of the poles are displayed in Fig. 13. The $\bar{P}_{b}$-pole is only present on the principal Riemann sheet for limited frequency range $818.75 \mathrm{kHz} \leq f \leq 2 \mathrm{MHz}$.

Allard et al. ${ }^{18}$ also found one of the poles and refer to it as an improper surface mode. Feng and Johnson ${ }^{7}$ stated that poles located to the left of shear-wave branch point $[\operatorname{Re}(p)$ $\left.<\operatorname{Re}\left(s_{S}\right)\right]$ have lost all physical significance as pseudo interface modes. In the latter paper, the authors consider pseudo interface modes in the conventional way. In their configuration, the additional poles might indeed lie on a different Riemann sheet, but we find that they can also show up on the principal Riemann sheet. From Fig. 4 we observe that the $\bar{P}_{a}$-pole has a substantial residue contribution to the full re- 
sponse, although it does not correspond to an interface wavemode $\left(\bar{P}_{b}\right.$-pole similarly). Any pole that contributes to the full response should be considered as physically significant.

Gilbert and Laster ${ }^{38}$ and Aki and Richards ${ }^{4}$ related the $\bar{P}$-poles in elastic solids to a separate arrival. Van der Hijden, ${ }^{17}$ however, stated that the concept of a separate pulse should be dismissed because it is just a peculiar tail to the compressional head-wave arrival. Harris and Achenbach ${ }^{36}$ confirmed this by stating that the poles yield features of the lateral waves. The observations in the current computations for poroelastic media also confirm this. From Fig. 7, we observe that the $\bar{P}_{a}$-pole contributes to the head waves generated by the $P_{1}$-wavefront. It also contributes to the $P_{1}$-wavefront itself because it yields a strong pulse that arrives even earlier (Figs. 4 and 7), which is obviously explained by the pole lying to the left of the compressionalwave slowness. The same is true for the $\bar{P}_{b}$-pole. The earlyarriving parts are not present in the full responses and hence, the $P_{1}$-waveform is constituted by both the residues of the $\bar{P}$-poles and the loop integrals along the branch cuts. The fact that a pole contributes to the $P_{1}$-waveform illustrates that it lies in the vicinity of the saddle point of the body wave, as used in asymptotic ray theory. ${ }^{35,39}$

There is one remarkable difference between the $\bar{P}$-poles in elastic and the ones in poroelastic media. In former, the poles never show up on the principal Riemann sheet ${ }^{4}$ while this is possible for the latter. A similarity lies in the fact that in elastic solids (with rather small values of Poisson's ratio), the poles lie also to the left of the compressional-wave slowness. $^{35}$

\section{CONCLUSIONS}

In this paper, we analyze the three-dimensional transient response of a fluid/poroelastic-medium configuration that is subjected to a vertical point force at the interface. For different materials, we quantitatively compare the full transient response with the residue contributions of pole singularities present on the so-called principal or physical Riemann sheet of integration. The poles are formed by zeroes of the poroelastic Stoneley dispersion equation, i.e., the pseudoStoneley $(p S t)$ and the pseudo-Rayleigh $(p R)$ poles.

We find that the residues of these poles do not necessarily contain all pertinent physical properties of the corresponding pseudo interface waves. Part of them can be captured by the loop integrals along the branch cuts. Therefore, it can be erroneous to use only the location of a zero of the Stoneley dispersion equation on the principal Riemann sheet, to predict the entire waveform, the propagation velocity, and attenuation of the corresponding pseudo interface wave.

According to the generally accepted explanation about the origin of a pseudo interface wave, it originates from a pole that lies on a nonprincipal Riemann sheet. The influence of the pole is only indirect in the sense that it causes a local maximum in the integrand of the Green's function when its location is close to the real axis. We find, however, that this conventional explanation is not necessarily confirmed in the context of Biot's theory for poroelasticity. The poles can
TABLE III. Symbols used in Appendix A The various indices are defined as $j=\left\{P_{1}, P_{2}, F, S\right\}, k=\left\{P_{1}, P_{2}\right\}$, and $\ell=\left\{P_{1}, P_{2}, S\right\}$. Behind a number of definitions, references are displayed where the specific expressions originate from.

\begin{tabular}{|c|c|c|}
\hline$\hat{\rho}_{11}$ & $=\rho_{11}-i \hat{b} / \omega$ & Ref. 23 \\
\hline$\hat{\rho}_{22}$ & $=\rho_{22}-i \hat{b} / \omega$ & Ref. 23 \\
\hline$\hat{\rho}_{12}$ & $=\rho_{12}+i \hat{b} / \omega$ & Ref. 23 \\
\hline$d_{0}$ & $=\hat{\rho}_{11} \hat{\rho}_{22}-\hat{\rho}_{12}^{2}$ & Ref. 23 \\
\hline$d_{1}$ & $=-\left(R \hat{\rho}_{11}+P \hat{\rho}_{22}-2 Q \hat{\rho}_{12}\right)$ & Ref. 23 \\
\hline$d_{2}$ & $=P R-Q^{2}$ & Ref. 23 \\
\hline$s_{k}^{2}$ & $=\left(-d_{1} \mp\left(d_{1}^{2}-4 d_{0} d_{2}\right)^{1 / 2}\right) /\left(2 d_{2}\right), \quad \operatorname{Im}\left(s_{k}\right) \leq 0$ & Ref. 23 \\
\hline$s_{S}^{2}$ & $=d_{0} /\left(G \hat{\rho}_{22}\right), \operatorname{Im}\left(s_{S}\right) \leq 0$ & Ref. 23 \\
\hline$s_{F}^{2}$ & $=\rho_{F} / K_{F}$ & \\
\hline$p$ & $=\left(p_{1}^{2}+p_{2}^{2}\right)^{1 / 2} \geq 0$ & \\
\hline$q_{j}$ & $=\left(s_{j}^{2}-p^{2}\right)^{1 / 2}, \quad \operatorname{Im}\left(q_{j}\right) \leq 0$ & \\
\hline$\hat{\beta}_{k}$ & $=-\left(\hat{\rho}_{11}-P s_{k}^{2}\right) /\left(\hat{\rho}_{12}-Q s_{k}^{2}\right)$ & Ref. 23 \\
\hline$\hat{\beta}_{S}$ & $=-\hat{\rho}_{12} / \hat{\rho}_{22}$ & Ref. 23 \\
\hline$A^{\prime}$ & $=A-(1-\phi) Q / \phi$ & Ref. 12 \\
\hline$Q^{\prime}$ & $=Q-(1-\phi) R / \phi$ & Ref. 12 \\
\hline$H_{k}$ & $=Q+R \hat{\beta}_{k}$ & Ref. 12 \\
\hline$K_{k}$ & $=A^{\prime}+Q^{\prime} \hat{\beta}_{k}+2 G$ & Ref. 12 \\
\hline$\phi_{\ell}$ & $=1-\phi+\phi \hat{\beta}_{\ell}$ & Ref. 12 \\
\hline$\Delta_{1}$ & $=s_{P 2}^{2} H_{P 2}-s_{P 1}^{2} H_{P 1}$ & \\
\hline$\Delta_{2}$ & $=q_{P 1} s_{P 2}^{2} H_{P 2}-q_{P 2} s_{P 1}^{2} H_{P 1}$ & \\
\hline$\Delta_{3}$ & $\begin{aligned}= & -4 p^{4} \phi \hat{\rho}_{22}^{-1}\left(q_{P 1} s_{P 1}^{2} H_{P 1}-q_{P 2} s_{P 2}^{2} H_{P 2}\right)+4 p^{2} q_{S} q_{P 1} q_{P 2} \phi \hat{\rho}_{22}^{-1} \Delta_{1} \\
& +2 p^{2} s_{S}^{2}\left(q_{P 1}\left(\phi_{P 1}+\phi d_{2}^{-1} H_{P 1} K_{P 2}\right)-q_{P 2}\left(\phi_{P 2}+\phi d_{2}^{-1} H_{P 2} K_{P 1}\right)\right) \\
& -s_{S}^{2} G^{-1}\left(q_{P 1} \phi_{P 1} s_{P 2}^{2} K_{P 2}-q_{P 2} \phi_{P 2} s_{P 1}^{2} K_{P 1}\right)\end{aligned}$ & \\
\hline
\end{tabular}

show up on the principal Riemann sheet. For the pSt-pole, we even show that its presence on the principal Riemann sheet is not only determined by the contrast in the material properties, but also by frequency.

Finally, we find that two additional zeroes of the poroelastic Stoneley dispersion equation do have physical significance due to their residue contributions to the fast compressional wavefront and to the head waves that are radiated by this wavefront. In the literature the additional poles are, however, referred to as nonphysical because they are not related to pseudo interface waves. The poles are comparable with the $\bar{P}$-poles known in nonporous elastic solids, Refs. 38 and 4. Depending on the specific material parameters and frequency, they can be present on the principal Riemann sheet or on another one.

\section{ACKNOWLEDGMENTS}

This research is supported by The Netherlands Research Centre for Integrated Solid Earth Sciences (ISES). The authors are also grateful to E. C. Slob and A. V. Metrikine for the valuable discussions, and to B. Rossen for textual remarks and suggestions.

\section{APPENDIX A: TRANSFORM-DOMAIN RESPONSE}

In this Appendix, we derive the $\left(\mathbf{p}, x_{3}, \omega\right)$-domain solution to Eqs. (5), (6), and (9)-(14). Many of the involved symbols are explained in Table III.

The general solution to the acoustic wave equation [Eq. (9)] in the $\left(\mathbf{p}, x_{3}, \omega\right)$-domain can be readily found by applying the Fourier transform [Eqs. (15) and (16)] and solving the obtained ordinary differential equation. The result is 


$$
\tilde{p}_{F}=i \omega \rho_{F} \widetilde{A}_{F} \exp \left(+i \omega q_{F} x_{3}\right), \quad x_{3}<0,
$$

where $\widetilde{A}_{F}$ is the complex plain-wave amplitude of the fluid $(F)$ wave and $q_{F}=\left(s_{F}^{2}-p^{2}\right)^{1 / 2}$ is the vertical slowness. It contains the wave slowness $s_{F}$ and the magnitude of the horizontal slowness $p$ that are defined in Table III.

The general solution to the Biot equations [Eqs. (5) and (6)] can be derived by applying Helmholtz decomposition in the $(\mathbf{x}, \omega)$-domain to these equations, according to ${ }^{12,23}$

$$
\begin{aligned}
& \hat{\mathbf{u}}=\nabla \hat{\varphi}_{P 1}+\nabla \hat{\varphi}_{P 2}+\nabla \times \hat{\boldsymbol{\psi}}, \\
& \hat{\mathbf{U}}=\hat{\beta}_{P 1} \nabla \hat{\varphi}_{P 1}+\hat{\beta}_{P 2} \nabla \hat{\varphi}_{P 2}+\hat{\beta}_{S} \nabla \times \hat{\boldsymbol{\psi}},
\end{aligned}
$$

where $\hat{\varphi}_{P 1}$ and $\hat{\varphi}_{P 2}$ denote the scalar potentials for the fast $\left(P_{1}\right)$ and slow $\left(P_{2}\right)$ compressional waves, respectively, and $\hat{\psi}$ denotes the shear-wave $(S)$ vector potential. $\hat{\beta}_{P 1}, \hat{\beta}_{P 2}$, and $\hat{\beta}_{S}$ are the well-known fluid-solid (frame) amplitude ratios ${ }^{23}$ for the separate body wavemodes (Table III).

Applying the Helmholtz decomposition, the governing equations are decoupled and once the spatial Fourier transform [Eq. (16)] is applied, the decoupled equations turn into ordinary differential equations for $\widetilde{\varphi}_{P 1}$ and $\widetilde{\varphi}_{P 2}$, and $\widetilde{\psi}$ that can be solved separately. The general solution for the displacements is obtained by adding the separate contributions according to Eqs. (A2) and (A3). When the shear-wave term is split into a vertically polarized $(S V)$ and a horizontally polarized $(S H)$ part, the result for the wave vector $\widetilde{\mathbf{w}}$ $=\left(\widetilde{u}_{1}, \widetilde{u}_{2}, \widetilde{u}_{3},-\phi \widetilde{p}_{f}\right)^{T}$ can be written as

$$
\begin{aligned}
\widetilde{\mathbf{w}} & =\left[\begin{array}{cccc}
p_{1} & p_{1} & q_{S} \frac{p_{1}}{p} & s_{S}^{2} \frac{p_{2}}{p^{2}} \\
p_{2} & p_{2} & q_{S} \frac{p_{2}}{p} & -s_{S}^{2} \frac{p_{1}}{p^{2}} \\
q_{P 1} & q_{P 2} & -p & 0 \\
-i \omega s_{P 1}^{2} H_{P 1} & -i \omega s_{P 2}^{2} H_{P 2} & 0 & 0
\end{array}\right] \\
& \times\left[\begin{array}{c}
\tilde{A}_{P 1} \exp \left(-i \omega q_{P 1} x_{3}\right) \\
\tilde{A}_{P 2} \exp \left(-i \omega q_{P 2} x_{3}\right) \\
\tilde{A}_{S V} \exp \left(-i \omega q_{S} x_{3}\right) \\
\tilde{A}_{S H} \exp \left(-i \omega q_{S} x_{3}\right)
\end{array}\right], \quad x_{3}>0 .
\end{aligned}
$$

Next to the solid displacements $\widetilde{\mathbf{u}}$, the wave vector $\widetilde{\mathbf{w}}$ contains the fluid pressure $\widetilde{p}_{f}$ rather then the fluid displacements $\widetilde{\mathbf{U}}$ because the four components of $\widetilde{\mathbf{w}}$ describe the wave field totally: there are only four independent variables, see Ref. 40. In Eq. (A4), $\widetilde{A}_{P 1}, \widetilde{A}_{P 2}, \widetilde{A}_{S V}$, and $\widetilde{A}_{S H}$ denote the complex plain-wave amplitudes of the corresponding body wavemodes. In Table III, the vertical slownesses $q_{P 1}, q_{P 2}$, and $q_{S}$ are defined (together with $q_{F}$ ), as well as the fluid compressibility terms $H_{P 1}$ and $H_{P 2}$.

The body-wave slownesses have $\operatorname{Im}\left(s_{j}\right) \leq 0$ and Sommerfeld's radiation condition requires that $\operatorname{Im}\left(q_{j}\right) \leq 0$ for all body modes, $j=\left\{P_{1}, P_{2}, F, S\right\}$.
The complex plane-wave amplitudes are determined by the boundary conditions at the interface $x_{3}=0$. Applying the transforms [Eqs. (15) and (16)] to the boundary conditions [Eqs. (10)-(14)] and substituting the wave fields [Eqs. (A1) and (A4)], the following set of equations is obtained

$$
\begin{gathered}
{\left[\begin{array}{ccccc}
2 G p^{2}-s_{P 1}^{2} K_{P 1} & 2 G p^{2}-s_{P 2}^{2} K_{P 2} & 0 & 2 G p q_{S} & 0 \\
s_{P 1}^{2} H_{P 1} & s_{P 2}^{2} H_{P 2} & -\phi \rho_{F} & 0 & 0 \\
q_{P 1} \phi_{P 1} & q_{P 2} \phi_{P 2} & q_{F} & -p \phi_{S} & 0 \\
2 p q_{P 1} & 2 p q_{P 2} & 0 & s_{S}^{2}-2 p^{2} & +\frac{p_{2}}{p_{1} p} q_{S} s_{S}^{2} \\
2 p q_{P 1} & 2 p q_{P 2} & 0 & s_{S}^{2}-2 p^{2} & -\frac{p_{1}}{p_{2} p} q_{S} s_{S}^{2}
\end{array}\right]} \\
\times\left[\begin{array}{c}
\tilde{A}_{P 1} \\
\widetilde{A}_{P 2} \\
\tilde{A}_{F} \\
\tilde{A}_{S V} \\
\widetilde{A}_{S H}
\end{array}\right]=\left[\begin{array}{c}
\hat{F} \\
\frac{i \omega}{\omega} \\
0 \\
0 \\
0
\end{array}\right],
\end{gathered}
$$

which is similar to that in Ref. 12, but extended to three dimensions. The constrained moduli $K_{P 1}$ and $K_{P 2}$ are defined in Table III. The solution is calculated analytically using MAPLE $^{\odot}$ :

$$
\begin{aligned}
\tilde{A}_{P 1}= & \frac{-\hat{F}}{i \omega G \Delta_{1} \Delta_{S t}}\left(\phi \rho_{F} q_{P 2}\left(s_{S}^{2} \phi_{P 2}-2 p^{2} \phi \hat{\rho}_{22}^{-1} s_{P 2}^{2} H_{P 2}\right)\right. \\
& \left.+q_{F}\left(s_{S}^{2}-2 p^{2}\right) s_{P 2}^{2} H_{P 2}\right), \\
\tilde{A}_{P 2}= & \frac{\hat{F}}{i \omega G \Delta_{1} \Delta_{S t}}\left(\phi \rho_{F} q_{P 1}\left(s_{S}^{2} \phi_{P 1}-2 p^{2} \phi \hat{\rho}_{22}^{-1} s_{P 1}^{2} H_{P 1}\right)\right. \\
& \left.+q_{F}\left(s_{S}^{2}-2 p^{2}\right) s_{P 1}^{2} H_{P 1}\right), \\
\tilde{A}_{F}= & \frac{\hat{F}}{i \omega G \Delta_{1} \Delta_{S t}}\left(\left(q_{P 1} \phi_{P 1} s_{P 2}^{2} H_{P 2}-q_{P 2} \phi_{P 2} s_{P 1}^{2} H_{P 1}\right)\right. \\
& \left.\times\left(s_{S}^{2}-2 p^{2}\right)+2 p^{2} \Delta_{2} \phi_{S}\right), \\
\tilde{A}_{S V}= & \frac{2 p \hat{F}}{i \omega G \Delta_{1} \Delta_{S t}}\left(\phi^{2} \rho_{F} \hat{\rho}_{22}^{-1} q_{P 1} q_{P 2} \Delta_{1}+q_{F} \Delta_{2}\right),
\end{aligned}
$$

and $\widetilde{A}_{S H}=0$. Here, the "poroelastic Stoneley-wave denominator" (see Sec. III) is defined as

$$
\Delta_{S t}=q_{F} \Delta_{R}+\phi \rho_{F} \Delta_{3} / \Delta_{1},
$$

which is associated with interface waves along the fluid/ poroelastic-medium interface. It is very similar to the "Scholte-wave denominator" for a fluid/elastic-solid interface, ${ }^{27}$ and equivalent to the one as given by Denneman et $a .^{41}$ It contains the "poroelastic Rayleigh-wave denominator" that is associated with interface waves along a vacuum/poroelastic-medium interface

$$
\Delta_{R}=\left(s_{S}^{2}-2 p^{2}\right)^{2}+4 p^{2} q_{S} \Delta_{2} / \Delta_{1},
$$

which is very similar to the one for a vacuum/elastic-solid interface. ${ }^{4,31}$ 
Now the plain-wave amplitudes are known, the $\left(\mathbf{p}, x_{3}, \omega\right)$-domain solution to Eqs. (5), (6), and (9)-(14) is determined and given by Eqs. (A1) and (A4).

\section{APPENDIX B: INVERSE FOURIER INTEGRAL}

In this Appendix, we show how Eq. (19) can be written in terms of a single integral according to Eq. (20), following Ref. 4. Transforming Eq. (19) to cylindrical coordinates according to $p_{1}=p \cos \varphi, p_{2}=p \sin \varphi$, and $x_{1}=r \cos \vartheta, x_{2}$ $=r \sin \vartheta$, where $r=\left(x_{1}^{2}+x_{2}^{2}\right)^{1 / 2}$, it can be written as

$$
\begin{aligned}
\hat{\mathbf{g}}^{+}= & \frac{\omega^{2}}{(2 \pi)^{2}} \int_{0}^{\infty} \int_{0}^{2 \pi} \frac{\widetilde{\mathbf{n}}^{+}\left(p, \varphi, x_{3}, \omega\right)}{\Delta_{S t}} \\
& \times \exp (-i \omega p r \cos (\varphi-\vartheta)) p d \varphi d p .
\end{aligned}
$$

The $\varphi$-dependence of $\widetilde{\mathbf{n}}^{+}$can be replaced by (horizontal) partial-derivative operators $\partial_{\alpha}, \alpha=\{1,2\}$, since the factors $p_{\alpha}$ that appear in $\widetilde{\mathbf{n}}^{+}$[see Eqs. (A4) and (17)] correspond to horizontal derivatives $\left(-i \omega p_{\alpha} \leftrightarrow \partial_{\alpha}\right)$ in the $(\mathbf{x}, \omega)$-domain. Therefore, $\widetilde{\mathbf{n}}^{+}\left(p, \varphi, x_{3}, \omega\right)$ is defined such that it contains the appropriate derivative operators according to

$$
\begin{aligned}
\hat{\mathbf{g}}^{+}= & \frac{\omega^{2}}{(2 \pi)^{2}} \int_{0}^{\infty} \frac{\widetilde{\mathbf{n}}^{+}\left(p, \partial_{\alpha}, x_{3}, \omega\right)}{\Delta_{S t}} \\
& \times \int_{0}^{2 \pi} \exp (-i \omega p r \cos (\varphi-\vartheta)) d \varphi p d p \\
= & \frac{\omega^{2}}{2 \pi} \int_{0}^{\infty} \frac{\widetilde{\mathbf{n}}^{+}\left(p, \partial_{\alpha}, x_{3}, \omega\right)}{\Delta_{S t}} J_{0}(\omega p r) p d p,
\end{aligned}
$$

where we used the integral representation of the zeroth-order Bessel function $J_{0}(\cdots)$, see Ref. 42. The Bessel function is replaced by the sum of two zeroth-order Hankel functions of the first and second kinds, ${ }^{42}$ i.e., $J_{0}(z)=\frac{1}{2}\left(H_{0}^{(1)}(z)+H_{0}^{(2)}(z)\right)$. Using the equality $H_{0}^{(1)}(z)=-H_{0}^{(2)}(-z)$ and the evenness of the $\left(\mathbf{p}, x_{3}, \omega\right)$-domain Green's functions in $p$, Eq. (B2) can be written as

$$
\hat{\mathbf{g}}^{+}=\frac{\omega^{2}}{4 \pi} \int_{-\infty}^{\infty} \frac{\widetilde{\mathbf{n}}^{+}\left(p, \partial_{\alpha}, x_{3}, \omega\right)}{\Delta_{S t}} H_{0}^{(2)}(\omega p r) p d p
$$

where the horizontal derivatives are applied to the Hankel function before the integration is performed, according to

$$
\partial_{\alpha} H_{0}^{(2)}(\omega p r)=-\omega p \frac{x_{\alpha}}{r} H_{1}^{(2)}(\omega p r) .
$$

${ }^{1}$ D. R. Burns, "Acoustic waveform logs and the in-situ measurement of permeability-A review," in Geophysical Applications for Geotechnical Investigations, edited by F. L. Paillet and W. T. Saunders (ASTM, Philadelphia, 1990).

${ }^{2}$ I. A. Viktorov, Rayleigh and Lamb Waves: Physical Theory and Applications (Plenum, New York, 1967).

${ }^{3}$ W. M. Ewing, W. S. Jardetzky, and F. Press, Elastic Waves in Layered Media (McGraw-Hill, New York, 1957).

${ }^{4}$ K. Aki and P. G. Richards, Quantitative Seismology (Freeman and Co., New York, 1980).

${ }^{5}$ J. H. Rosenbaum, "Synthetic microseismograms: Logging in porous formations," Geophysics 39, 14-32 (1974).

${ }^{6} \mathrm{H}$. Deresiewicz, "The effect of boundaries on wave propagation in a liquid-filled porous solid: IV. Surface waves in a half-space," Bull. Seismol. Soc. Am. 52, 627-638 (1962).
${ }^{7}$ S. Feng and D. L. Johnson, "High-frequency acoustic properties of a fluid/ porous solid interface. I. New surface mode," J. Acoust. Soc. Am. 74, 906-914 (1983).

${ }^{8}$ S. Feng and D. L. Johnson, "High-frequency acoustic properties of a fluid/ porous solid interface. II. The 2D reflection Green's function,” J. Acoust. Soc. Am. 74, 915-924 (1983).

${ }^{9}$ M. J. Mayes, P. B. Nagy, L. Adler, B. P. Bonner, and R. Streit, "Excitation of surface waves of different modes at fluid-porous solid interface," J. Acoust. Soc. Am. 79, 249-252 (1986).

${ }^{10}$ L. Adler and P. B. Nagy, "Measurements of acoustic surface waves on fluid-filled porous rocks," J. Geophys. Res. 99, (B9), 17863-17869 (1994).

${ }^{11}$ J. F. Allard, M. Henry, C. Glorieux, W. Lauriks, and S. Petillon, "Laserinduced surface modes at water-elastic and poroelastic interfaces," J. Appl. Phys. 95, 528-535 (2004).

${ }^{12}$ A. A. Gubaidullin, O. Y. Kuchugurina, D. M. J. Smeulders, and C. J. Wisse, "Frequency-dependent acoustic properties of a fluid/porous solid interface," J. Acoust. Soc. Am. 116, 1474-1480 (2004).

${ }^{13}$ D. L. Johnson, J. Koplik, and R. Dashen, "Theory of dynamic permeability and tortuosity in fluid-saturated porous-media," J. Fluid Mech. 176, 379-402 (1987).

${ }^{14}$ I. Edelman and K. Wilmanski, "Asymptotic analysis of surface waves at vacuum/porous medium and liquid/porous medium interfaces," Continuum Mech. Thermodyn. 14, 25-44 (2002).

${ }^{15} \mathrm{~B}$. Albers, "Monochromatic surface waves at the interface between poroelastic and fluid half-spaces," Proc. R. Soc. London, Ser. A 462, 701723 (2006).

${ }^{16}$ M. G. Markov, "Low-frequency Stoneley wave propagation at the interface of two porous half-spaces," Geophys. J. Int. 177, 603-608 (2009).

${ }^{17}$ J. H. M. T. van der Hijden, "Quantitative analysis of the pseudo-Rayleigh phenomenon," J. Acoust. Soc. Am. 75, 1041-1047 (1984).

${ }^{18}$ J. F. Allard, G. Jansens, G. Vermeir, and W. Lauriks, "Frame-borne surface waves in air-saturated porous media," J. Acoust. Soc. Am. 111, 690-696 (2002).

${ }^{19}$ J. F. Allard, M. Henry, C. Glorieux, S. Petillon, and W. Lauriks, "Laserinduced surface modes at an air-porous medium interface," J. Appl. Phys. 93, 1298-1304 (2003).

${ }^{20} \mathrm{M}$. A. Biot, "Theory of propagation of elastic waves in a fluid-saturated porous solid. I. Low-frequency range," J. Acoust. Soc. Am. 28, 168-178 (1956).

${ }^{21}$ M. A. Biot, "Theory of propagation of elastic waves in a fluid-saturated porous solid. II. Higher frequency range," J. Acoust. Soc. Am. 28, 179191 (1956).

${ }^{22}$ M. A. Biot and D. G. Willis, "The elastic coefficients of the theory of consolidation,” J. Appl. Mech. 24, 594-601 (1957).

${ }^{23}$ J. F. Allard, Propagation of Sound in Porous Media: Modelling of Sound Absorbing Materials (Elsevier Applied Science, London, 1993).

${ }^{24}$ D. M. J. Smeulders, R. L. G. M. Eggels, and M. E. H. van Dongen, "Dynamic permeability: Reformulation of theory and new experimental and numerical data," J. Fluid Mech. 245, 211-227 (1992).

${ }^{25}$ H. Deresiewicz and R. Skalak, "On uniqueness in dynamic poroelasticity," Bull. Seismol. Soc. Am. 53, 783-788 (1963).

${ }^{26}$ B. Gurevich and M. Schoenberg, "Interface conditions for Biot's equations of poroelasticity,” J. Acoust. Soc. Am. 105, 2585-2589 (1999).

${ }^{27}$ A. T. de Hoop and J. H. M. T. van der Hijden, "Generation of acoustic waves by an impulsive line source in a fluid-solid configuration with a plane boundary," J. Acoust. Soc. Am. 74, 333-342 (1983).

${ }^{28}$ K. Attenborough, S. I. Hayek, and J. M. Lawther, "Propagation of sound above a porous half-space," J. Acoust. Soc. Am. 68, 1493-1501 (1980).

${ }^{29}$ M. Abramowitz and I. A. Stegun, Handbook of Mathematical Functions (Dover, New York, 1972).

${ }^{30}$ B. A. Fuchs, B. V. Shabat, and J. Berry, Functions of a Complex Variable and Some of Their Applications (Pergamon, Oxford, 1964).

${ }^{31}$ J. D. Achenbach, Wave Propagation in Elastic Solids (North-Holland, Amsterdam, 1973).

${ }^{32}$ P. J. Davies and P. Rabinowitz, Methods of Numerical Integration (Academic, New York, 1975).

${ }^{33} \mathrm{~N}$. Ricker, "Wavelet contraction, wavelet expansion, and the control of seismic resolution," Geophysics 18, 769-792 (1953).

${ }^{34} \mathrm{C}$. J. Wisse, "On frequency dependence of acoustic waves in porous cylinders," Ph.D. thesis, Delft University of Technology, The Netherlands (1999).

${ }^{35} \mathrm{~L}$. Tsang, "Time-harmonic solution of the elastic head wave problem incorporating the influence of Rayleigh poles," J. Acoust. Soc. Am. 63, 1302-1309 (1978). 
${ }^{36}$ J. G. Harris and J. D. Achenbach, "Comment on 'On the complex conjugate roots of the Rayleigh equation: The leaky surface wave'," J. Acoust. Soc. Am. 112, 1747-1748 (2002).

${ }^{37}$ J. G. Scholte, "The range of existence of Rayleigh and Stoneley waves," Mon. Not. R. Astron. Soc. 5, 120-126 (1947).

${ }^{38}$ F. Gilbert and S. J. Laster, "Excitation and propagation of pulses on an interface," Bull. Seismol. Soc. Am. 52, 299-319 (1962).

${ }^{39}$ B. L. van der Waerden, "On the method of saddle points," Appl. Sci. Res.,
Sect. B 2, 33-45 (1952).

${ }^{40} \mathrm{G}$. Bonnet, "Basic singular solutions for a poroelastic medium in the dynamic range," J. Acoust. Soc. Am. 82, 1758-1762 (1987).

${ }^{41}$ A. I. M. Denneman, G. G. Drijkoningen, D. M. J. Smeulders, and K. Wapenaar, "Reflection and transmission of waves at a fluid/porousmedium interface," Geophysics 67, 282-291 (2002).

${ }^{42}$ I. S. Gradshteyn and I. M. Ryzhik, Table of Integrals, Series and Products (Academic, London, 1980). 